\title{
The Association between Periodontitis and Diabetes among Filipino Dental School Patients: A Retrospective Case-control Study
}

\author{
Ma. Celina U. Garcia, DDM and Zen Alfred B. Nemenzo \\ Department of Clinical Dental Health Sciences, College of Dentistry, University of the Philippines Manila
}

\begin{abstract}
Objective. The association between periodontitis and diabetes mellitus varies based on geographical location, and there are limited investigations on the relationship of the two chronic diseases among Philippine subjects. This study aimed to determine the association between periodontitis and diabetes among Filipino dental school patients.

Methods. In this retrospective case-control study, the periodontal conditions and diabetic statuses of all patients who sought treatment at the Dental College's Oral Medicine clinic within two academic years were reviewed. The prevalence of diabetes among 715 cases of periodontitis was compared to the prevalence among 834 control patients without periodontitis. Odds ratios (OR) were computed using logistic regression analysis, adjusting for age, sex, educational attainment, and smoking status.
\end{abstract}

Results. The overall prevalence of diabetes mellitus for all patients was $3.10 \%$. Patients with periodontitis had a significantly higher prevalence of diabetes at $5.73 \%$ compared to control subjects without periodontitis, whose diabetes prevalence was recorded at $0.84 \%(P<0.001)$. A significant association was observed between periodontitis and diabetes, with an unadjusted $\mathrm{OR}=7.19$. After logistic regression analysis to control confounding variables, the adjusted OR was found to be 3.05 (95\% confidence interval = 1.27 to $7.31 ; P=0.012$ ).

Conclusion. The prevalence and odds of having diabetes are significantly higher among Filipino dental school patients with periodontitis, compared to patients without periodontitis. These results provide evidence to support an association between periodontitis and diabetes mellitus in this representative sample of the Filipino population.

Keywords: periodontitis, diabetes mellitus, Philippines, association, prevalence

\section{INTRODUCTION}

The global burden of diabetes mellitus is rising to epidemic levels, with 463 million estimated cases in 2019 accounting for $9.3 \%$ of the world's adult population aged 20-79. The International Diabetes Federation expects these numbers to rise to more than 700 million cases by $2045 .^{1}$ Periodontal disease is another chronic disease cited as the most common chronic inflammatory condition of humans worldwide, with a global prevalence of 50\% among adults. ${ }^{2}$ Moreover, a recent review has reported that severe periodontitis affects approximately $10 \%$ of the world's population. ${ }^{3}$ In the Philippines, $6.3 \%$ or over 3.9 million of the adult population are affected by diabetes, while $48 \%$ of the Filipino population has a form of periodontal disease. ${ }^{1,4}$

The bidirectional relationship between diabetes mellitus and periodontal disease has been extensively studied and reviewed. Evidence has established that diabetes is a risk factor for periodontitis and that hyperglycemia affects 
periodontal outcomes. ${ }^{2,5}$ Hence, diabetic patients may have a higher severity of periodontal disease. ${ }^{6}$ A summary of evidence has led to the recognition of periodontal disease as the sixth complication of diabetes. ${ }^{7}$ Meanwhile, the role of periodontitis on possible diabetes complications and incident diabetes remains less clear. New evidence suggests that periodontal disease adversely affects glycemic control and that individuals with periodontitis have an increased risk for developing type II diabetes. ${ }^{8,9}$ However, some studies point to no association between periodontitis and the risk for diabetes. ${ }^{10}$

Numerous studies have been conducted to look into the association between diabetes and periodontitis, and in the past decade, the observed association between the two chronic diseases has varied among different populations. In a recent systematic review, the prevalence of diabetes ranged from as low as $2.3 \%$ to as high as $36 \%$ among populations from Asia, Europe, North and South America. Moreover, the overall odds of being afflicted with diabetes was higher among individuals with periodontitis as compared to those without periodontitis. ${ }^{11}$ An investigation on the prevalence and association of various systemic diseases, including diabetes mellitus, to periodontitis among Swedish dental school patients likewise showed an association between the two diseases, with $21.2 \%$ of periodontitis patients presenting with diabetes compared to only $6.7 \%$ in the control group without periodontitis. ${ }^{12}$ On the other hand, studies in Austrian and Korean populations did not find any association between diabetes and periodontitis after adjustment for confounding variables. ${ }^{13,14}$ Moreover, although an association between diabetes and periodontitis was observed in a New Zealand population, the relationship between the two chronic diseases was dependent on the periodontitis case definition used..$^{15}$

The results of the aforementioned researches suggest that aside from methodological differences affecting the outcome of studies, the presence and strength of the association between diabetes and periodontitis may vary based on geographical distribution. Therefore, it has been suggested that additional studies be conducted globally in the majority of countries. ${ }^{11}$

The contribution of Philippine data to the current pool of knowledge on the diabetes-periodontitis relationship is sparse. Findings from earlier researches suggest that the prevalence of periodontitis is high among Filipino type II diabetes mellitus patients, with a $68.23 \%$ prevalence reported by Bitong et al. and a 100\% prevalence rate based on a study by Dolor et al. ${ }^{16,17}$ To the authors' knowledge, there is no previous case-control study that has investigated the association between diabetes and periodontitis in a Filipino population, and there remains a need for recent data and studies that identify and describe such an association in the Philippine setting. Thus, this present study aimed to determine the association between periodontitis and diabetes mellitus among Filipinos, with patients seeking periodontal treatment at the College of Dentistry of the University of the Philippines Manila as the representative population.

\section{MATERIALS AND METHODS}

The protocol for this retrospective case-control study through a review of dental and medical records was approved by the University of the Philippines Manila Research Ethics Board and complied with the Philippine Data Privacy Act of 2012. ${ }^{18}$ No personal information that may identify patients was collected from the records.

\section{Data Source and Study Population}

A total census of all dental charts of Filipino patients who sought periodontal treatment at the Oral Medicine Section Clinic of the College of Dentistry, the University of the Philippines Manila within academic years 2016-2017 and 2017-2018 was performed.

The minimum required sample size to find an association between periodontitis and diabetes mellitus is 610 . This was computed using $\mathrm{G}$ power 3.1.9.2 sample size calculation for logistic regression. An odds ratio of 2.95 was used based on the findings by Marjanovic and Buhlin as the association between periodontitis and diabetes. ${ }^{12}$ The probability of periodontitis patients having diabetes mellitus was set at 0.50 since it would yield the highest sample size. The level of significance and power were set at 0.05 and 0.8 respectively.

All patients who seek dental treatment at the College clinic initially undergo medical and dental history taking, oral diagnosis, and treatment planning, with information recorded in an Admitting Section (AS) chart. Those patients assessed as needing periodontal treatment based on the initial screening examination are referred to the Oral Medicine Section, where the results of a full mouth examination and a periodontal diagnosis are written down in a periodontal chart.

A total of 1,937 patients sought periodontal consult and treatment at the College within the two academic years that constituted the study period. The AS and periodontal charts of these patients were initially screened. Included were all patients who were Filipinos and aged 18 and above. Out of the 1,937 patients, 388 were excluded from the study due to the following criteria: (1) below 18 years of age $(n=151),(2)$ corresponding AS charts could not be retrieved ( $n=136)$, (3) medical histories were not updated within the study period $(n=100)$, and (4) chart was not signed by the attending faculty member $(n=1)$. The final number of included charts for data analysis was 1,549 (Figure 1).

Further manual review of AS and periodontal charts of all included patients was performed. Data in the AS charts include patients' demographics and medical history. The charts had been filled out by dental student clinicians through the patient interview, and the results of history taking and clinical examination in each chart were corroborated by dental faculty members. In the present study, information collected from the AS chart included each patient's age, 


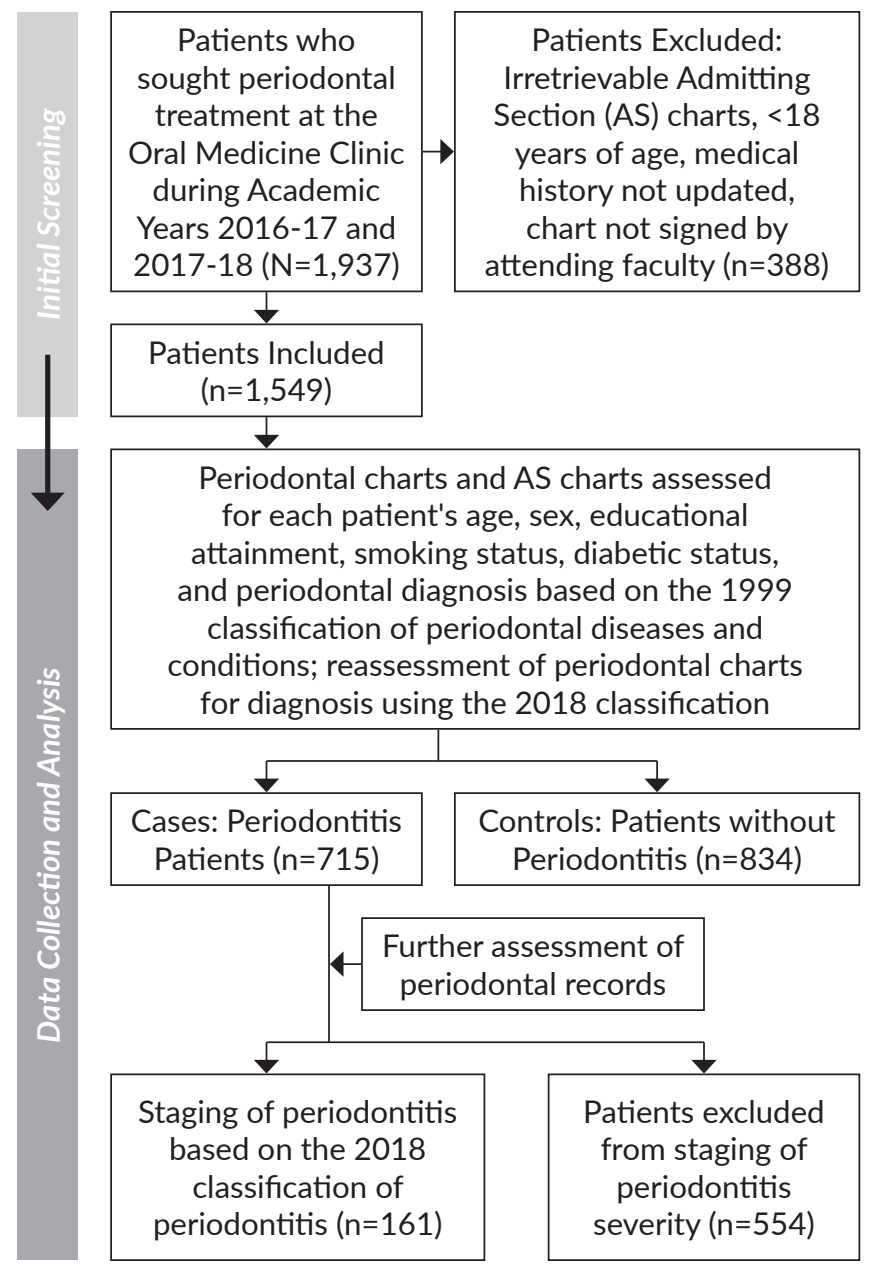

Figure 1. Flow diagram of chart screening for inclusion in the study's data analysis.

sex, educational attainment, smoking status, and diabetic status. Patients classified as having diabetes included those who self-reported having been diagnosed by a physician, those who were taking hypoglycemic agents or insulin, and patients with attached documents confirming a diagnosis of diabetes such as a physician's note, fasting plasma glucose, or HbA1c laboratory results. For smoking status, non-smokers were patients who either claimed to have never smoked or previous smokers who had stopped at least 5 years since the time of oral diagnosis. On the other hand, patients classified as smokers were those who self-reported to smoke regularly or occasionally or had stopped smoking less than 5 years.

Periodontal charts were then reviewed for the diagnosis given after a full-mouth periodontal assessment. Periodontal examinations were previously conducted by student clinicians, and the results of the examination along with the indicated diagnosis were confirmed by one of the eight periodontology faculty members of the college. Diagnosis at the time of initial examination was based on the 1999 classification of periodontal diseases. ${ }^{19}$ However, given the update in the classification of periodontal and peri-implant diseases and conditions in 2018, the initial diagnoses were reassessed following the new classification. ${ }^{20}$ Of the 1,549 patients who were included in the study, 715 had periodontitis and were designated as cases. None of the patients had clinical gingival health and the remaining 834 had received a diagnosis of gingivitis and constituted the control group without periodontitis (Figure 1).

Further assessment of the periodontal charts of the 715 periodontitis cases was performed, to determine the severity of periodontal destruction for staging based on the 2018 classification of periodontitis. Periodontitis staging was done to compare periodontal disease severity between those with and without diabetes mellitus. During their first appointment for a periodontal examination, patients normally undergo a full-mouth assessment, which includes a recording of sites with bleeding and pockets. However, clinical attachment levels (CAL) are recorded only for key teeth. Only those patients who consent to further periodontal management at the college undergo a subsequent examination with the recording of CAL on 6 sites of all teeth. Out of the 715 cases of periodontitis, 161 patients had periodontal records with sufficient data to retrospectively stage the periodontitis based on the 2018 classification of periodontal and peri-implant diseases and conditions. ${ }^{21,22}$ Patients with stage I and II periodontitis were designated as having mild and moderate periodontitis, respectively. On the other hand, those classified as falling under stages III and IV were all designated as severe periodontitis patients.

\section{Statistical Analysis}

The prevalence of diabetes mellitus among patients with periodontitis (cases) and patients without periodontitis (controls) was determined. The demographic variables (sex, age group, educational attainment, and smoking status) of cases and controls were described using frequency distribution, and comparison of these categorical variables for significant differences between cases and controls was determined through Fisher's exact or Chi-square test, for two and more than two categories respectively. An odds ratio analysis was performed to determine the initial association (unadjusted odds ratio) between periodontitis and diabetes mellitus among Filipino patients. A logistic regression model for multivariate analysis was set at a $95 \%$ confidence interval and used to control for identified confounding variables, namely age, sex, educational attainment, and smoking status. The logistic regression analysis yielded the final adjusted odds ratio for the association between periodontitis and diabetes. All biostatistical computations were performed using STATA version 14 software, at a 0.05 significance level.

\section{RESULTS}

\section{Patient Characteristics}

Of the 1,549 included patients, 715 were diagnosed with periodontitis and were classified as cases, while 834 were 
Table 1. Comparison of patient characteristics between periodontitis cases and control patients without periodontitis

\begin{tabular}{|c|c|c|c|}
\hline Characteristic & $\begin{array}{c}\text { With } \\
\text { periodontitis } \\
n(\%)\end{array}$ & $\begin{array}{c}\text { Without } \\
\text { periodontitis } \\
n(\%)\end{array}$ & $P$-value \\
\hline \multicolumn{4}{|l|}{ Age $(n=1,549)$} \\
\hline $\begin{array}{l}18-20 \\
21-30 \\
31-40 \\
41-50 \\
51-60 \\
61-70 \\
>70 \\
\end{array}$ & $\begin{array}{c}24(3.36 \%) \\
106(14.83 \%) \\
158(22.10 \%) \\
189(26.43 \%) \\
133(18.60 \%) \\
91(12.72 \%) \\
14(1.96 \%) \\
\end{array}$ & $\begin{array}{c}204(24.46 \%) \\
430(51.56 \%) \\
96(11.51 \%) \\
55(6.59 \%) \\
34(4.08 \%) \\
13(1.56 \%) \\
2(0.23 \%) \\
\end{array}$ & $<0.0001$ \\
\hline \multicolumn{4}{|l|}{$\operatorname{Sex}(n=1,549)$} \\
\hline $\begin{array}{l}\text { Male } \\
\text { Female }\end{array}$ & $\begin{array}{l}281(39.30 \%) \\
434(60.70 \%)\end{array}$ & $\begin{array}{l}300(35.71 \%) \\
534(64.29 \%)\end{array}$ & 0.188 \\
\hline \multicolumn{4}{|c|}{ Educational attainment $(n=1,412)$} \\
\hline $\begin{array}{l}\text { No schooling } \\
\text { Elementary } \\
\text { High school } \\
\text { Vocational } \\
\text { College } \\
\text { Postgraduate }\end{array}$ & $\begin{array}{c}2(0.31 \%) \\
44(6.72 \%) \\
237(36.18 \%) \\
6(0.92 \%) \\
363(55.42 \%) \\
3(0.46 \%) \\
\end{array}$ & $\begin{array}{c}0 \text { (0.0\%) } \\
17(2.25 \%) \\
208(27.48 \%) \\
7(0.92 \%) \\
513(67.77 \%) \\
12(1.59 \%) \\
\end{array}$ & $<0.0001$ \\
\hline \multicolumn{4}{|c|}{ Smoking status ( $n=1,547)$} \\
\hline $\begin{array}{l}\text { Smokers } \\
\text { Non-smokers }\end{array}$ & $\begin{array}{l}162(22.66 \%) \\
553(77.34 \%)\end{array}$ & $\begin{array}{l}131(15.75 \%) \\
701(84.25 \%) \\
\end{array}$ & 0.001 \\
\hline
\end{tabular}

diagnosed with either generalized or localized gingivitis, and were classified under the control group. Patient characteristics of both cases and controls are shown in Table 1 .

The majority of all the patients included in the analysis belonged to the 21 to 30-year-old age group (34.6\%). Among periodontitis cases, the majority $(59.71 \%)$ were $>40$ years of age. Those belonging to the $41-50$ age group constituted the highest percentage (26.43\%) among the cases. More than half of the patients in the control group were aged 21-30 (51.56\%) and the lowest percentage among controls belonged to those aged $>70$ years old $(0.23 \%)$. There were more females than males in both cases and controls, but with no significant statistical difference between groups $(P=0.188)$.

Data on educational attainment were available for 1,412 patients. Patients who reached at least college level comprised $62.04 \%$ of the total population. This was followed by high school level (31.52\%), elementary level (4.32\%), postgraduate level (1.06\%), vocational courses $(0.92 \%)$, and patients without schooling (0.14\%). College level was the predominant educational attainment level for both cases and controls (55.42\% and $67.77 \%$ respectively).

Data on smoking status were available for 1,547 patients, $18.94 \%$ of whom claimed to be smokers. On the other hand, $75.50 \%$ had never smoked cigarettes, while $5.56 \%$ were previous smokers who had stopped smoking at least 5 years before consulting. Among periodontitis patients, smokers accounted for $22.66 \%$ which was significantly higher than the control patients where only $15.75 \%$ claimed to be smokers $(P=0.001)$.
Table 2. Frequency and prevalence of diabetes mellitus among cases and controls

\begin{tabular}{lcc}
\multicolumn{1}{c}{ Diabetic Status } & $\begin{array}{c}\text { Periodontitis } \\
\text { Cases } \\
(\mathbf{n}=\mathbf{7 1 5})\end{array}$ & $\begin{array}{c}\text { Without } \\
\text { Periodontitis } \\
(\mathbf{n}=\mathbf{8 3 4})\end{array}$ \\
\hline With diabetes mellitus & $41(5.73 \%)$ & $7(0.84 \%)$ \\
Without diabetes mellitus & $674(94.27 \%)$ & $827(99.16 \%)$ \\
\hline
\end{tabular}

Table 3. Distribution of periodontitis severity among patients with and without diabetes mellitus

\begin{tabular}{lccc} 
& \multicolumn{3}{c}{ Periodontitis Severity } \\
\cline { 2 - 4 } \multicolumn{1}{c}{ Diabetic Status } & $\begin{array}{c}\text { Mild } \\
(\mathbf{n}=\mathbf{1})\end{array}$ & $\begin{array}{c}\text { Moderate } \\
(\mathbf{n}=\mathbf{8})\end{array}$ & $\begin{array}{c}\text { Severe } \\
(\mathbf{n}=\mathbf{1 5 2})\end{array}$ \\
\hline With diabetes mellitus & 0 & 0 & 15 \\
Without diabetes mellitus & 1 & 8 & 137 \\
\hline
\end{tabular}

\section{Comparison of the Prevalence of Diabetes Mellitus Between Cases and Controls}

The overall prevalence of diabetes mellitus for all patients included in the data analysis was $3.10 \%$. Patients with periodontitis had a significantly higher prevalence of diabetes at $5.73 \%$ compared to patients without periodontitis, whose diabetes prevalence was recorded at $0.84 \%(P<0.001$; Table 2).

\section{Comparison of Periodontitis Severity Between Diabetic and Nondiabetic Patients}

Among the 715 cases of periodontitis, 161 patients had sufficient data in their periodontal charts for staging under the 2018 case definition for periodontitis..$^{21,22}$ Of these, 152 were categorized as severe cases of periodontitis (Stages III and IV), eight cases as moderate periodontitis (Stage II), and one case as mild periodontitis (Stage I). The frequency distribution of the three severity groups among patients with and without diabetes can be seen in Table 3. None of the patients with mild and moderate periodontitis had diabetes, while $9.87 \%$ of all patients with severe periodontitis had diabetes. Given that diabetes was present in only 15 periodontitis cases, the data collected was insufficient to be able to draw a statistical correlation between the presence of diabetes and severity of periodontitis with the desired statistical power. However, all patients who had both diabetes and periodontitis were found to have severe periodontal destruction.

\section{Odds Ratios Between Periodontitis and Diabetes Mellitus}

Data analysis demonstrated a significant association between periodontitis and diabetes mellitus, with an unadjusted odds ratio (OR) of 7.19 (Table 4). Patients aged greater than 50 years old were highly associated with periodontitis $(\mathrm{OR}=7.99)$, while those whose educational attainment was college level or higher had lower odds of having periodontitis $(\mathrm{OR}=0.56)$. Moreover, cigarette smoking 
Table 4. Unadjusted odds ratios (OR) and adjusted odds ratios (AOR) for the association of periodontitis with diabetes and confounding variables

\begin{tabular}{|c|c|c|c|c|}
\hline \multirow{2}{*}{ Variable } & \multicolumn{2}{|c|}{ Unadjusted } & \multicolumn{2}{|l|}{ Adjusted } \\
\hline & OR $(95 \% \mathrm{Cl})$ & P-value & AOR $(95 \% \mathrm{CI})$ & P-value \\
\hline Diabetes mellitus & 7.19 (3.16-19.08) & $<0.001$ & $3.05(1.27-7.31)$ & 0.012 \\
\hline Age $(>50)$ & $7.99(5.72-11.33)$ & $<0.001$ & $7.90(5.52-11.33)$ & $<0.0001$ \\
\hline Sex (Female) & $0.87(0.70-1.07)$ & 0.188 & $0.79(0.62-1.01)$ & 0.056 \\
\hline Educational attainment (College level or higher) & $0.56(0.45-0.70)$ & $<0.001$ & $0.54(0.43-0.69)$ & $<0.0001$ \\
\hline Smoker & $1.57(1.20-2.04)$ & $<0.001$ & $1.49(1.10-2.03)$ & 0.01 \\
\hline
\end{tabular}

was found to be significantly associated with periodontitis $(\mathrm{OR}=1.57)$. After logistic regression analysis to control confounders, a significant association between periodontitis and diabetes mellitus was still identified, with an adjusted odds ratio (AOR) of 3.05 (CI 1.27-7.31; $P=0.012$; Table 4).

\section{DISCUSSION}

This study examines the association between periodontitis and diabetes mellitus in a representative Filipino population consisting of patients who sought periodontal consult at a Philippine dental college. The overall diabetes prevalence was $3.10 \%$, which is lower compared to both the national (6.3\%) and global (9.3\%) prevalence rates reported by the International Diabetes Federation. ${ }^{1}$ However, it is important to note that the findings of this present study may not be representative of the general Filipino population due to sampling being limited to periodontal patients at the University within two academic years.

This present study demonstrated that $5.73 \%$ of Filipino patients with periodontitis had diabetes, compared to only $0.84 \%$ in control patients without periodontitis $(P<0.001)$. To the authors' knowledge, there is no previous case-control study that has analyzed the association between periodontitis and diabetes in a Filipino population. However, in a study by Vergel de Dios, Murjani, and Garcia, the researchers identified the prevalence of specified systemic diseases, which included diabetes mellitus, among periodontitis patients from two private periodontal practices and the same University dental clinic as this present study. The overall prevalence of diabetes was $3.46 \%$, but a lower prevalence $(2.16 \%)$ was observed among periodontitis patients from the University clinic when compared to private practice patients (3.58\%). The authors stated that patient characteristics and cultural practices inherent to the Philippine setting may account for the non-detection of medical conditions and the relatively lower prevalence of diabetes in their study. Patients seeking treatment at the University usually belong to the lower socio-economic strata, and access to regular medical checkups is limited. The authors further inferred that patients at the University may also advertently withhold their medical conditions for fear that disclosure may result in deferment of dental treatment, and patients will be forced to secure medical clearance requiring out-of-pocket expenses. ${ }^{23}$
Prevalence rates of diabetes among periodontitis patients are variable across different nationality groups as seen in other researches. In a recent systematic review by Ziukate, Slot, and Van der Weijden, an analysis of 27 studies revealed an overall weighted mean diabetes prevalence of $13.1 \%$, with prevalence ranging from $2.3 \%$ to $36 \%$ among populations from Asia, Europe, North and South America. ${ }^{11}$ The authors also reported that variation in prevalence was geographically related, with the lowest prevalence observed in studies with European populations. On the other hand, periodontitis patients in Asian countries were noted to have the highest prevalence of diabetes at $17.2 \%$, which is higher than the $5.73 \%$ diabetes prevalence recorded in this present study. However, differences in periodontitis case definitions used by the studies in the systematic review preclude direct comparison with the present study. Moreover, methods for determining the presence of diabetes also varied. In the present study, the majority of the diabetic patients were identified through self-reporting during the patient interview, with some individuals having presented a physician's note or laboratory test confirming the diagnosis. This method may therefore have affected the outcome of the present study. In sub-analyses by Ziukate et al., studies that determined the presence of diabetes through clinical testing yielded a higher prevalence of diabetes among periodontitis patients (17.3\%) as opposed to patients who self-reported their condition either through a questionnaire, interview, or claimed use of hypoglycemic agents (6.2\%).

A retrospective case-control study by Sperr et al. involved periodontitis patients from an Austrian dental school. Compared with our present study, a relatively similar prevalence of diabetes mellitus (5.9\%) was observed in the Austrian cases of periodontitis. However, the authors reported no significant difference in comparison to their control cohort (6.1\%), unlike in our present study's control group which was found to have a significantly lower prevalence compared to cases. The study of Sperr et al. differs in that the control group was selected from the general Austrian population, and cases were age and sex-matched, with two control individuals assigned for each periodontitis case. ${ }^{13}$ On the other hand, in another retrospective case-control study, this time in a Swedish population, a significantly higher prevalence of diabetes was likewise observed among periodontitis patients (21.2\%) as opposed to periodontally 
healthy controls $(6.7 \%) .{ }^{12}$ However, the reported prevalence for both groups were much higher than the prevalence of diabetes that was detected in the present study. As with the present study, cases and controls were also unmatched, and the Swedish population also consisted of patients who sought treatment at a dental school. However, individuals comprising the Swedish population were relatively older, with periodontitis patients having a mean age of 61.7 years. In the present study, only $14.68 \%$ of cases were 61 years and older. Among different age groups, older adults have been found to have the highest prevalence of diabetes, with diabetes onset reported to be usually in the $4^{\text {th }}$ or $5^{\text {th }}$ decade of life. Moreover, the incidence of diabetes is said to have an increasing trend with increasing age until about 65 years of age. ${ }^{24,25}$ Similarly, the prevalence and severity of periodontitis has been reported to increase with age and may be attributed to the chronic nature of the disease, as well as to the additive effects of long-term exposure to etiologic and true risk factors. ${ }^{26}$ The aforementioned age-related findings on diabetes and periodontitis may explain the higher prevalence of diabetes among periodontitis cases compared to the control group in this present study, given that among cases, the majority were older than 40 years, while most of the controls were $\leq 40$ years of age.

This study also compared the severity of periodontitis between patients with and without diabetes mellitus. Among the 715 periodontitis cases, only $22.52 \% \quad(n=161)$ had periodontal records with sufficient data for staging under the 2018 case definition of periodontitis severity. ${ }^{21,22}$ During their first appointment for a periodontal assessment, patients at the dental college normally undergo a full mouth examination, which includes a recording of sites with bleeding and pockets. However, clinical attachment levels (CAL) are usually not recorded for all sites during this initial visit. Only those patients who proceed with further periodontal treatment at the college undergo a subsequent examination with the recording of CAL on 6 sites of all teeth. Given that periodontitis staging could only be determined for a limited number of patients, and diabetes was present in only 15 of the 161 periodontitis cases, the data collected was insufficient to be able to draw a statistical correlation between the presence of diabetes mellitus and the severity of periodontitis with the desired statistical power. However, a key finding in this study is that all patients who had both diabetes and periodontitis were found to have severe periodontal destruction. In a study among Fijian periodontitis patients seen in a dental school clinic, $16.0 \%$ had diabetes, and among the 55 patients with diabetes, more than half $(50.9 \%)$ had severe periodontitis, while $36.4 \%$ and $12.7 \%$ had moderate and mild periodontitis, respectively. However, although the authors reported having reviewed dental charts with Community Periodontal Index scores and periodontitis severity records, the severity classification used was not indicated. ${ }^{27}$ In contrast, no association was found between diabetes and severe periodontitis in a matched case-control study in a
New Zealand population, wherein cases were matched with a control group (without severe periodontitis) in terms of age group, sex, and socioeconomic status. After adjusting for smoking exposure, ethnicity, dental visit pattern, and number of teeth, conditional logistic regression modeling did not find any association using five different case definitions for severe periodontitis. ${ }^{15}$

In the present study, an association between periodontitis and diabetes was found among Filipino patients, with an unadjusted odds ratio of 7.19. When adjusted for age, sex, educational attainment, and smoking status through a logistic regression analysis, a significant association remained, with an adjusted odds ratio of 3.05. This significant association is consistent with other studies linking diabetes mellitus as a risk factor for periodontitis. In the case-control study in a Swedish population, an association between diabetes and periodontitis was reported, with a comparable albeit slightly lower adjusted odds ratio of 2.95 in contrast to the present study. ${ }^{12}$ As with the Swedish population study, the present study controlled for age, sex, and smoking status. However, in addition to the aforementioned confounding variables, the present study also considered educational attainment in the regression analysis. Socioeconomic status indicators, which include education, are inversely related to the prevalence and severity of the periodontal disease, with higher educational levels associated with lower prevalence and odds of having periodontitis. ${ }^{28} \mathrm{~A}$ significant correlation was also revealed in the systematic review by Ziukate et al. Based on the analysis, the overall odds ratio was 2.27 for diabetes mellitus patients to be among periodontitis subjects as compared to individuals without periodontitis. ${ }^{11}$

On the contrary, the Austrian study by Sperr et al. reported lower odds for diabetes mellitus to be associated with periodontitis. The unadjusted odds ratio was 0.87 , and after adjustment for confounding variables through a logistic regression analysis, an adjusted $\mathrm{OR}$ of 0.55 $(P<0.001)$ was reported. ${ }^{13}$ Similar to the present study, the Austrian population study controlled for sex, age, educational attainment, and smoking status. However, additional variables (alcohol consumption and BMI) were considered in the analysis by Sperr et al. On the other hand, no association was found in the study that examined the records of a Korean population. Upon multivariate logistic regression analysis to consider the interaction of smoking and diabetes, as well as to control confounders (age, sex. educational attainment, toothbrushing frequency, dental visits, and family income), the authors reported an adjusted odds ratio of $1.21(P=0.336){ }^{14}$ Similarly, in the analysis of data from the 2009 to 2012 National Health and Nutrition Survey, self-reported diabetes was not associated with periodontitis based on weighted multivariate analysis $(\mathrm{AOR}=0.90, P=0.40$ ), upon adjustment of covariates. However, while glycosylated hemoglobin cutoff levels of $7.0 \%$ and $7.5 \%$ were not associated, higher cutoff levels of $8.0 \%, 8.5 \%$, and $9.0 \%$ were significantly correlated with adjusted odds ratios of having periodontitis. ${ }^{29}$ 
Certain limitations of this present study must be considered when interpreting the results. As mentioned earlier, the findings of this study cannot be applied to the general Filipino population due to sampling being restricted to Filipino patients who sought dental treatment at the college. Another study limitation is that multiple student clinicians performed history taking and periodontal assessment. Although a standard form (AS chart) was used for assessing systemic conditions and symptoms during the patient interview, and the filled-up charts were corroborated by attending faculty, variations in questioning may have affected the accuracy of medical information gathered. The periodontal assessment and diagnosis were likewise performed by different student clinicians. However, as with the AS charts, periodontal charts were verified by faculty members. Moreover, the 161 charts with full mouth recording of CAL were verified by faculty who are certified by the Philippine Board of Periodontology. The inherent limitations of self-reported medical conditions also apply to this study. There remains a possibility of recall bias and under-reporting of diabetes mellitus and other medical conditions, especially when patients do not know that they have the disease in question. However, several studies have previously shown the validity of self-reporting medical conditions. In particular, a moderate to high sensitivity (55-95\%) and generally high specificity (84-99\%) was observed in patients who claimed to have diabetes. ${ }^{30-33}$

\section{CONCLUSION}

Data from the present study support the association of periodontitis and diabetes mellitus among Filipinos, with patients seeking periodontal treatment at a Philippine dental college as the representative population. The significantly higher prevalence and odds of having diabetes among periodontitis cases compared with those without periodontitis corroborate previous research findings that diabetes may be a risk factor for periodontitis. Additional epidemiologic studies using a nationally representative Filipino adult population, with the matching of cases and controls in terms of age, gender, socioeconomic level, and smoking status are recommended.

\section{Acknowledgments}

The authors would like to thank Drs. Jessica RebuenoSantos, Maricar Lachica, and Michelle Sunico-Segarra, for their technical advice in the conduct of the study, and Ms. Caren Bacsid for performing the statistical analysis.

\section{Statement of Authorship}

Both authors contributed to the conception and design of the study, and data acquisition, analysis, and interpretation. Both authors prepared the draft, while the first author critically revised the manuscript. Both authors have approved the version to be published and have agreed to be accountable for all aspects of the work, ensuring integrity and accuracy.

\section{Author Disclosure}

The authors declared that they have no conflicts of interest concerning this study.

\section{Funding Source}

This research was supported in part by a grant from the National Institutes of Health, University of the Philippines Manila.

\section{REFERENCES}

1. International Diabetes Federation, IDF Diabetes Atlas 9th ed [Internet]. 2019 [cited 2021 Jan]. Available from: https://www. diabetesatlas.org/en/resources/.

2. Chapple ILC, Genco R. Diabetes and periodontal diseases: consensus report of the joint EFP/AAP workshop on periodontitis and systemic diseases. J Clin Periodontol. 2013 Apr;40(Suppl. 14):S106-12.

3. Frencken JE, Sharma P, Stenhouse L, Green D, Laverty D, Dietrich T. Global epidemiology of dental caries and severe periodontitis - a comprehensive review. J Clin Periodontol. 2017 Mar;44(Suppl. 18):S94-S105.

4. Department of Health. National Monitoring and Evaluation Dental Survey. 2011.

5. Mealey BL, Oates TW. Diabetes mellitus and periodontal diseases. J Periodontol. 2006 Aug;77(8):1289-303.

6. Khader YS, Dauod AS, El-Qaderi SS, Alkafajei A, Batayha WQ. Periodontal status of diabetics compared with nondiabetics: a metaanalysis. J Diabetes Complications. 2006 Jan-Feb;20(1):59-68.

7. Löe H. Periodontal disease. The sixth complication of diabetes mellitus. Diabetes Care. 1993 Jan;16(1):329-34.

8. Borgnakke WS, Ylöstalo PV, Taylor GW, Genco RJ. Effect of periodontal disease on diabetes: systematic review of epidemiologic observational evidence. J Periodontol. 2013 Apr;84(Suppl 4):S135-52.

9. Graziani F, Gennai S, Solini A, Petrini M. A systematic review and meta-analysis of epidemiologic observational evidence on the effect of periodontitis on diabetes. J Clin Periodontol. 2018 Feb;45(2):167-87.

10. Joshipura KJ, Muñoz-Torres FJ, Dye BA, Leroux BG, Ramírez-Vick M, Pérez CM. Longitudinal association between periodontitis and development of diabetes. Diabetes Res Clin Pract. 2018 Jul;141: 284-93.

11. Ziukaite L, Slot DE, Van der Weijden FA. Prevalence of diabetes mellitus in people clinically diagnosed with periodontitis: A systematic review and meta-analysis of epidemiologic studies. J Clin Periodontol. 2018 Jun;45(6):650-62.

12. Marjanovic M, Buhlin, K. Periodontal and systemic diseases among Swedish dental school patients- a retrospective register study. Oral Health Prev Dent. 2013;11(1):49-55.

13. Sperr M, Kundi M, Tursic V, Bristela M, Moritz A, Andrukhov O, et al. Prevalence of comorbidities in periodontitis patients compared with the general Austrian population. J Periodontol. 2018 Jan; 89:19-27.

14. Han DH, Lim S, Kim JB. The association of smoking and diabetes with periodontitis in a Korean population. J Periodontol. 2012 Nov;83(11):1397-1406.

15. Knight ET, Leichter JW, Tawse-Smith A, Thomson WM. Quantifying the association between self-reported diabetes and periodontitis in the New Zealand population. J Periodontol. 2015 Aug;86(8):945-54.

16. Bitong ED, Jasul GVJ, Dellosa MAG. Prevalence of periodontitis and its association with glycemic control among patients with type 2 diabetes mellitus seen at St. Luke's Medical Center. Philipp J Intern Med. 2010 Jan-Jun;48(1):9-14.

17. Dolor MFB, Estaniel TMD, Garcia MCU. A descriptive study on the prevalence and severity of periodontitis among Filipino type II diabetic patients at UP-PGH-OPD, Section of Endocrinology, Diabetes and Metabolism [thesis]. Manila, PH: University of the Philippines Manila; 2009. 
18. National Privacy Commission. Republic Act 10173 - Data Privacy Act of 2012. Available from: https://www.privacy.gov.ph/data-privacy-act/.

19. Armitage GC. Development of a classification system for periodontal diseases and conditions. Ann Periodontol. 1999 Dec;4(1):1-6.

20. Caton JG, Armitage G, Berglundh T, Chapple ILC, Jepsen S, Kornman $\mathrm{KS}$, et al. A new classification scheme for periodontal and peri-implant diseases and conditions - Introduction and key changes from the 1999 classification. J Clin Periodontol. 2018 Jun;45 Suppl 20:S1-S8.

21. Tonetti MS, Greenwell H, Kornman, KS. Staging and grading of periodontitis: Framework and proposal of a new classification and case definition. J Periodontol. 2018 Jun;89(Suppl 1):S159-S172.

22. Papapanou PN, Sanz M, Buduneli N, Dietrich T, Feres M, Fine DH, et al. Periodontitis: Consensus report of workgroup 2 of the 2017 World Workshop on the Classification of Periodontal and PeriImplant Diseases and Conditions. J Clin Periodontol. 2018 Jun;45 (Suppl 20):S162-S170.

23. Vergel de Dios NV, Murjani BV, Garcia MCU. Prevalence of systemic diseases among periodontal patients at the State University clinic and two private periodontal practices in the Philippines. In: Bartold PM, Ishikawa I, Zhang J, editors. A perspective of periodontal systemic relationships for the Asian Pacific region. Adelaide: Asian Pacific Society of Periodontology; 2008. p. 69-76.

24. Kirkman MS, Briscoe VJ, Clark N, Florez H, Haas LB, Halter JB, et al. Diabetes in older adults. Diabetes Care. 2012 Dec;35(12):2650-64.

25. Casanova L, Hughes FJ, Preshaw PM. Diabetes and periodontal disease: a two-way relationship. Br Dent J. 2014 Oct;217(8):433-7.
26. Papapanou PN, Lindhe J. Epidemiology of periodontal diseases. In: Lindhe JL, Lang NP, editors. Clinical periodontology and implant dentistry. Volume 1. 6th ed. Oxford: Wiley Blackwell; 2015. p.125-166.

27. Thomas A, Maimanuku LR, Mohammadnezhad M, Khan S. Presence and types of systemic diseases among patients with periodontitis in Suva, Fiji. J Healthc Commun. 2018;3(2):22.

28. Borrell LN, Crawford ND. Socioeconomic position indicators and periodontitis: examining the evidence. Periodontol 2000. 2012 Feb;58(1):69-83.

29. Garcia D, Tarima S, Okunseri C. Periodontitis and glycemic control in diabetes: NHANES 2009 to 2012. J Periodontol. 2015 Apr;86(4): 499-506.

30. Martin LM, Leff M, Calonge N, Garrett C, Nelson DE. Validation of self-reported chronic conditions and health services in a managed care population. Am J Prev Med. 2000 Apr;18(3):215-18.

31. Schneider ALC, Pankow JS, Heiss G, Selvin E. Validity and reliability of self-reported diabetes in the Atherosclerosis Risk in Communities Study. Am J Epidemiol. 2012 Oct;176(8):738-43.

32. Goldman N, Lin IF, Weinstein M, Lin YH. Evaluating the quality of self-reports of hypertension and diabetes. J Clin Epidemiol. 2003 Feb;56(2):148-54.

33. Pastorino S, Richards M, Hardy R, Abington J, Wills A, Kuh D, et al. Validation of self-reported diagnosis of diabetes in the 1964 British birth cohort. Prim Care Diabetes. 2015 Oct;9(5):397-400. 